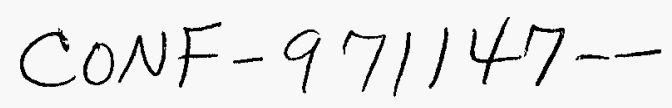

Author(s):

Submitted to:
Mohini W. Rawool-Sullivan

Robert J. Estep

David Miko

\section{IEEE Conference}

Albuquerque, NM

Nov. $10-14,1997$
Th

MAR 25500

(2)

\title{
MASTER
}

$\therefore$

DISTRIBUTION OF THIS DONUMENT IS UNLIMTEO

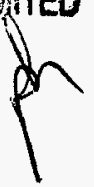

$\operatorname{tin} 60^{-n+3}$

\section{Los Alamos}

NATIONAL LABORATORY

Los Alamos National Laboratory, an affirmative action/equal opportunity employer, is operated by the University of California for the U.S. Department of Energy under contract W-7405-ENG-36. By acceptance of this article, the publisher recognizes that the U.S. Government retains a nonexclusive, royalty-free license to publish or reproduce the published form of this contribution, or to allow others to do so, for U.S. Government purposes. Los Alamos National Laboratory requests that the publisher identify this article as work performed under the auspices of the U.S. Department of Energy. The Los Alamos National Laboratory strongly supports academic freedom and a researcher's right to publish; as an institution, however, the Laboratory does not endorse the viewpoint of a publication or guarantee its technical correctness. 


\section{DISCLAIMER}

This report was prepared as an account of work sponsored by an agency of the United States Government. Neither the United States Government nor any agency thereof, nor any of their employees, makes any warranty, express or implied, or assumes any legal liability or responsibility for the accuracy, completeness, or usefulness of any information, apparatus, product, or process disclosed, or represents that its use would not infringe privately owned rights. Reference herein to any specific commercial product, process, or service by trade name, trademark, manufacturer, or otherwise does not necessarily constitute or imply its endorsement, recommendation, or favoring by the United States Government or any agency thereof. The views and opinions of authors expressed herein do not necessarily state or reflect those of the United States Government or-any agency thereof. 


\title{
Estimation of Obliquely Scattered Gamma-Ray Response Functions in the Gross- Count Tomographic Gamma Scanner (GC-TGS) Method
}

\author{
Mohini W. Rawool-Sullivan, Robert J. Estep, and David Miko \\ Los Alamos National Laboratory \\ Los Alamos, NM 87545
}

\begin{abstract}
It was recently shown that a logarithmic response-function technique based on the material basis set (MBS) formalism used in the tomographic gamma scanner (TGS) method allows gross spectra from $\mathrm{NaI}$ detectors to be used in both measuring MBS transmission corrections using external transmission sources and in applying the corrections to emission spectra to arrive at radionuclide mass estimates. In this work we have attempted to show that addition of the oblique scatter component can increase the accuracy of the GC-TGS measurements with both $\mathrm{NaI}$ and high-purity germanium detectors. This paper describes the formalism behind the GCTGS method and the improvement achieved in the analysis by including the scatter component.
\end{abstract}

\section{INTRODUCTION}

The gross-count tomographic gamma scanner (GC-TGS) method for assaying transuranic waste in 208-1 drums is being developed to allow $\mathrm{NaI}$ and other low-resolution gamma-ray detectors to be used in place of high-purity germanium (HPGe) detectors in TGS systems [1], [2]. In TGS assays, low-resolution transmission (attenuation coefficient) images of 208-1 drums are used to calculate attenuation corrections for subsequent emission-image reconstructions of the radionuclides within the drums (usually ${ }^{239} \mathrm{Pu}$ or ${ }^{235} \mathrm{U}$ ). With HPGe detectors, one uses continuum-subtracted full-energy peaks for the analysis. With NaI detectors, however, subtraction of the continuum is inaccurate and introduces large statistical errors. Ordinary response function fitting is not practical because the spectra involved are distorted by the drum matrix. However, as was demonstrated in [2] for simple geometries, a logarithmic response-function decomposition based on the material basis set (MBS) model [3] can be just as accurate as HPGe methods. Moreover, the application of the attenuation corrections to the emission spectra is nonsubtractive, so significant gains in sensitivity can be achieved. For this reason, the method is also useful when using HPGe detectors.

The application of the GC approach to transmission imaging has been found to work well, as predicted in [2] . In emission imaging, however, the scattering of gamma rays into the detector by the drum matrix (i.e., the nonradioactive materials inside the drum) constitutes a significant interference. In this paper we examine the magnitude and nature of these oblique scatter effects and present a simple single-scatter model for including them in the GC-TGS analysis. Section II provides a background discussion on the TGS and MBS methods and the gross-count method as it is applied to transmission and emission imaging. Section III presents experimental data taken to understand the nature of the obliquely scattered radiation. Section IV describes our model for including scatter effects in the GC-TGS method. Section V presents TGS scan data and results along with our conclusions.

\section{BACKGROUND}

\section{A. The TGS and MBS methods}

The emission imaging problem is formulated for $n_{\text {views }}$ tomographic views and $n_{\text {voxels }}$ image voxels as

$$
\mathbf{d}(e)=\mathrm{F}(e) \mathbf{s},
$$

where $\mathbf{d}(e)$ is an $n_{\text {vicws }}$-vector of detector count rates for gamma-rays of energy index $e, \mathbf{s}$ is the $n_{\text {voxels }}$-vector of radionuclide masses to be solved for, and $\mathrm{F}(e)$ is an $n_{\text {views }}$ by $n_{\text {voxels }}$ attenuation-corrected efficiency matrix. $\mathrm{F}(e)$ is obtained from an uncorrected, energy-independent efficiency matrix $E$ and the energy-dependent attenuation-coefficient image vector $\mu(e)$ through a direct application of Beer's law, i.e.,

$$
\begin{aligned}
& \mathrm{F}(e)_{\mathrm{ij}}=\mathrm{E}_{\mathrm{ij}} \mathrm{A}(e)_{\mathrm{ij}} c_{\mathrm{e}}, \\
& \mathrm{A}(e)_{\mathrm{ij}}=\prod_{\mathrm{k}} \exp \left\{-t_{\mathrm{ijk}} \mu(e)_{\mathrm{k}}\right\}
\end{aligned}
$$

where $c_{\mathrm{e}}$ is a calibration constant for the eth energy and $t_{\mathrm{j} \mathrm{k}}$ is the thickness of the $k$ th attenuating voxel in a line between the $j$ th emitting voxel and the detector in the $i$ th measurement position.

It is apparent that some method is required to convert $\mu$ vectors from transmission-source energies to emission-source energies, and also that calculating the triple loop implicit in (2) for a large number of emission energies would be prohibitively time consuming. We address both issues by using the material basis set (MBS) approach[3] which generalizes the dual-energy method of Engler et al.[4] to arbitrary numbers $n_{\mathrm{c}}$ of energies and $n_{\mathrm{z}}$ of basis materials. In the MBS we write the attenuation coefficient $\mu(e)_{\mathrm{j}}$ for the $j$ th voxel and $e$ th energy as a linear combination of the known attenuation coefficients $\mathrm{U}_{\mathrm{cr}}$ for the eth energy and $z$ th basis material as

$$
\mu(e)_{j}=\Sigma_{\mathrm{z}} \mathrm{U}_{\mathrm{ez}} \rho(z)_{\mathrm{j}} .
$$

The partial density $\rho(z)$, gives the fractional amount of the $z$ th basis material in the $j$ th voxel. Substituting in (2), we get for the attenuation factor

$$
\mathrm{A}(e)_{\mathrm{ij}}=\Pi_{\mathrm{z}} \exp \left\{-\mathrm{X}_{\mathrm{ij} \mathrm{z}, \mathrm{c} z} \mathrm{U}_{\mathrm{c},}\right\},
$$


where

$$
\mathrm{X}_{\mathrm{ijz}}=\Sigma_{\mathrm{k}} t_{\mathrm{ijk}} \rho(z)_{\mathrm{j}}
$$

This replaces $n_{\mathrm{e}}$ loops over the $n_{\text {voxels }}$ image voxels (indexed by $k$ ) with $n_{\mathrm{e}}$ loops over the $n_{\mathrm{z}}$ basis materials plus $n_{\mathrm{z}}$ loops over the image voxels.

Conversion between transmission and emission energy sets is almost automatic in the MBS method. One solves for the energy-independent transmission images $\rho_{z}$ using a matrix $U^{\prime}$ of attenuation coefficients for the transmission energies, then applies the $\rho_{2}$ vectors to the emission problem using another matrix $U$ specific to the emission energies but for the same basis materials. With an $n_{\mathrm{z}}$ of two or three materials the method is quite accurate over the energy region of 100 to $2000 \mathrm{keV}$, the region of most interest for assaying transuranicwaste drums. As has been shown[5], the linear formulation of the attenuation problem in (3) through (5) leads to three linear methods for coupling transmission scans taken at different energies: data-space coupling (our method of choice), imagespace coupling (not recommended), and full coupling into a single large system of equations (not significantly better than the much faster data-space coupling). In data-space coupling we solve for the aggregate partial density vectors $\rho_{i}^{*}$ at each tomographic view $(i)$, and then use the elements $\rho^{*}{ }_{z, i}$ to solve separately for the images $\rho_{z}$ using

$$
\rho^{*}{ }_{z, i}=\sum_{j} T_{i j} \rho_{z, j}
$$

or in matrix notation,

$$
\rho_{z}^{*}=\mathrm{T} \rho_{\mathrm{z}},
$$

where the matrix element $T_{i j}$ is the thickness of the $j$ th voxel along a line connecting the transmission source and the detector in the $i$ th tomographic view. $T$ is the same matrix used when reconstructing the usual attenuation-coefficient images from log-transmission measurements.

\section{B. The Gross-Count Method}

\section{1) Transmission imaging}

In the original TGS method the count rates $\mathbf{d}(e)$ in (1) referred to continuum-subtracted full-energy peaks measured with an HPGe detector. In GC transmission imaging, transmission spectra at each tomographic view are converted to log-transmission form and a constrained linear responsefunction decomposition is performed using log-transmission basis spectra measured through the set of $n_{z}$ basis materials. The coefficients from the decomposition are the aggregate partial densities, $\rho^{*}{ }_{2, j}$, which are subsequently used in equation (6) to solve for the MBS images $\rho_{z}$. These partial density data and image vectors are equivalent to those obtained using HPGe continuum-subtracted peaks, and can be used interchangeably in subsequent analyses. We have found GC transmission images produced from $\mathrm{NaI}$ detectors to be just as accurate as those obtained using HPGe continuum-subtracted peaks. Because tomographic transmission measurements involve a simple straight line source-absorber-detector geometry, no corrections are required for oblique scattering of gamma rays. As is discussed in another paper at this conference[6], the low-angle forward scattering that one sees is mostly accounted for in the basis spectra. This is not the case with emission imaging.

\section{2) Emission imaging}

The GC method for emission imaging makes use of a general rule for combining emission data when the solution vector $\mathbf{s}$ is the same for every energy (i.e., $\mathbf{s}=\mathbf{s}(e)=\mathbf{s}\left(e^{\prime}\right)$, for all $\left.e, e^{\prime}\right)$. Summing equation (1) over all $n_{\mathrm{c}}$ emission energies, one obtains

$$
\mathbf{d}=\mathrm{F} \mathbf{s},
$$

where

$$
\begin{aligned}
& \mathbf{d}=\sum_{\mathrm{c}} \mathbf{d}(e) \\
& \mathrm{F}=\Sigma_{c} \mathrm{~F}(e) .
\end{aligned}
$$

In $\mathrm{GC}$ emission imaging, the summed count rates $\mathbf{d}$ are the emission spectra summed over an appropriate channel range (e.g., for assaying ${ }^{239} \mathrm{Pu}$ we sum the spectra between approx. 100 and $500 \mathrm{keV}$ ). Only ambient background is subtracted; there is no continuum subtraction or response-function fitting performed on the emission data. For this reason, significantly better assay sensitivity is achieved than when using continuum-subtracted full-energy peaks.

The attenuation-corrected emission matrix $\mathrm{F}(e)$ is computed for each channel $e$ using equations (4) and (5). The speed advantage of the MBS approach becomes particularly apparent in this context, where a large number of energies can be involved, depending on the degree of spectrum compression used. Rather than using attenuation coefficients $\mathrm{U}_{\mathrm{e}}$, from tables, we use measured log-transmission response spectra $\mathbf{u}_{2}$ for a reference source transmitted through the materials in the basis set. The reference source must have the same isotopic composition and physical form as the radionuclide(s) being assayed, although the error introduced by slight differences will be negligible. The response spectra become columns in the emission U matrix, i.e.,

$$
\mathrm{U}_{e, \mathrm{z}} \equiv \mathrm{u}_{\mathrm{z}}(e)
$$

where $u_{z}(e)$ is the negative logarithm of the counts in channel $e$ of the spectrum through material $z$ divided by the unattenuated counts for the same source and channel number. Equation (4) then becomes

$$
\mathrm{A}(e)_{\mathrm{ij}}=\prod_{\mathrm{z}} \exp \left\{-\mathrm{X}_{\mathrm{ijz}} \mathbf{u}_{\mathrm{z}}(e)\right\}
$$

and the summed emission matrix $F$ is given by

$$
\mathrm{F}_{\mathrm{ij}}=\mathrm{E}_{\mathrm{ij}}\left\{\Sigma_{\mathrm{e}} c_{\mathrm{i}} \Pi_{z} \exp \left\{-\mathrm{X}_{\mathrm{ijz}} \mathrm{u}_{\mathrm{z}}(e)\right\}\right\} .
$$

We will refer to the matrix $F$ in (10) as the "scatter-free" emission matrix. What is missing from this model is the 
higher angle obliquely scattered gamma rays, which can scatter into the detector even when the emitting source is outside the detector's field of view. In the rest of this paper we describe the effects of this oblique scattering and present a model for including them in the TGS emission matrix.

\section{OBLIQUELY SCATTERED RADIATION}

The current TGS algorithms measure gamma rays emitted directly from a radiation and transmission source. In addition to this, in the GC method we take into account the gamma rays that are scattered off the matrix surrounding the source, and assume the matrix in question is not necessarily between the source and the detector. The radiation reaching the detector after a single scattering event is angle-dependent and can be described by the Klein-Nishima formula [7] as

$$
\mathrm{d} \sigma / \mathrm{d} \Omega=\left(\mathrm{r}_{\mathrm{e}}^{2} / 2\right) *\left(1 / \mathrm{C}^{2}\right) *\left\{1+\mathrm{B}+\left[\gamma^{2} \mathrm{~A}^{2} / \mathrm{C}\right]\right\} .
$$

Here $r_{e}$ is the classical electron radius, B is $\cos ^{2} \theta$, A is $(1-\cos \theta)$ and $C$ is $(1+\gamma A)$. The quantity $\gamma$ is given by $\gamma=h v / m_{c} c^{2}$, where $\mathrm{m}_{\mathrm{c}}$ is the electron mass, $\mathrm{c}$ is the velocity of light and $\mathrm{h} v$ is the energy of the incident gamma ray. Integration of this formula over $d \Omega$ gives the total probability per electron for Compton scattering to occur. This angular dependence of the singlescattering data was measured in our laboratory and is shown in Figure 1 .

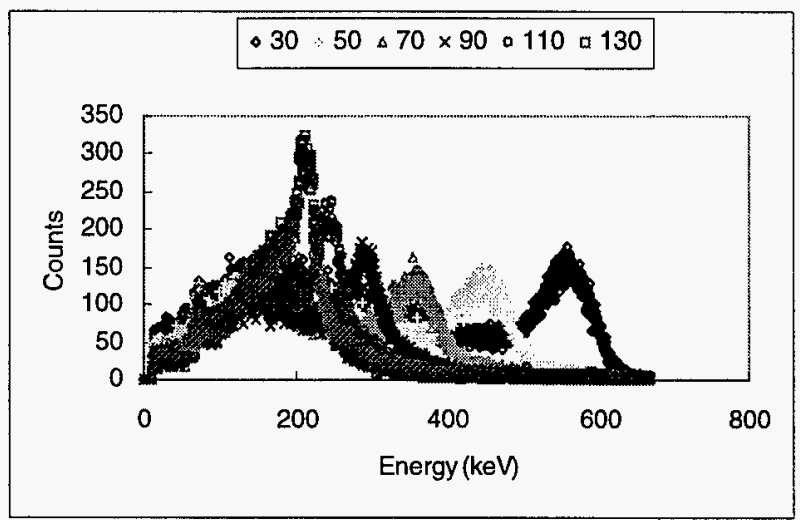

Figure 1: ${ }^{137} \mathrm{Cs}$ spectra for various values of $\theta$. The legend values show $\theta$ in units of degree.

Figure 1 shows the data taken using a $10-\mathrm{mCi},{ }^{137} \mathrm{Cs}$ source for various $\theta$ values, where $\theta$ is the angle between the incident and outgoing gamma rays. The data was taken using a 5.08cm-diameter and 5.08-cm-long NaI crystal for $\theta$ values between 30 and 130 degrees. The peaks in the spectra are from the photoelectric interaction in the detector of the Compton scattered gamma rays for the respective values of $\theta$. We have tabulated the peak centroids from these spectra in Table 1, along with the calculated value of outgoing gammaray energy $\left(h v^{\prime}\right)$ as a function of $\theta$ in degrees, using the kinematic equation

$$
h v^{\prime}=h v /(1+\gamma *(1-\cos \theta))
$$

As can be seen in the table, the agreement between the measured and calculated centroids is very good.

Table 1.

Predicted and calculated values of the photopeak of the Compton scattered gamma rays.

\begin{tabular}{|c|c|c|}
\hline$\theta$ (degrees) & $\begin{array}{c}\text { Calculated } \\
\text { Centroid }(\mathrm{keV})\end{array}$ & $\begin{array}{c}\text { Measured Centroid } \\
(\mathrm{keV})\end{array}$ \\
\hline 30 & 564 & $559.90 \pm 5$ \\
\hline 40 & 508 & $499.70 \pm 3$ \\
\hline 50 & 452 & $449.74 \pm 2$ \\
\hline 70 & 357 & $359.85 \pm 2$ \\
\hline 90 & 288 & $287.69 \pm 2$ \\
\hline 110 & 242 & $245.58 \pm 2$ \\
\hline 130 & 212 & $214.69 \pm 2$ \\
\hline
\end{tabular}

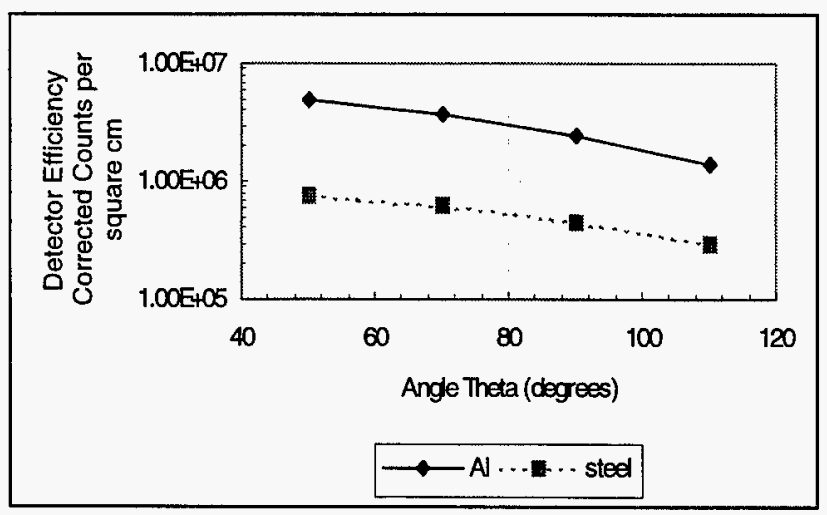

Figure 2: Difference in single scatter between $\mathrm{Al}$ and steel.

In Figure 2 we have shown the detector-efficiencycorrected counts per square area of the collimated gamma-ray source intercepted by the scatterer. Both data sets were taken for 150 seconds using a $10-\mathrm{mCi}{ }^{137} \mathrm{Cs}$ source. The background subtracted total counts in the region of interest (ROI) 100-800 $\mathrm{keV}$ were used. The difference in these counts mainly came from the difference in the attenuation of the incoming and outgoing gamma rays and the dependence of the Compton crosssection on the number of electrons present in the scatterer. The incoming and outgoing gamma rays suffer more attenuation in steel than in $\mathrm{Al}$. The $\mathrm{Z}$ is larger for steel than for the $\mathrm{Al}$, thus the Compton scattering cross-section for given angle is larger for steel than for Al. Figure 2 shows that the low- $Z$ material contributes more significantly to the continuum than the high- $Z$ material, as the attenuation effects dominate.

Besides the single-scattering effects discussed above, it is also possible to have multiple scattering effects. The scattering angles of radiation reaching the detector following multiple interactions are almost random. Thus the contribution from such events to the continuum is same no matter what the geometry between source and the scattering material is. 


\section{A MODEL FOR OBLIQUE SCATTERING IN GC-TGS}

The treatment of oblique scatter in the CG-TGS model assumes that the cross section as well as the shape and energy of the scattered spectrum are functions of the scattering angle, and that for a given angle the number of gamma rays scattered is proportional to the electron density within the scattering voxel. The attenuation within the scattering voxel, between the emitting and scattering voxels, and between the scattering voxel and the detector, along with relevant geometric efficiencies, are treated as separable effects. Figure 3 defines the geometry and some of the quantities used. In the figure, gamma-rays of energy index $e$ emitted from the $j$ th voxel scatter off the $k$ th voxel into the detector in the $i$ th view. The $k$ th voxel subtends a solid angle $4 \pi \mathrm{E}_{\mathrm{jk}}^{\prime}$ when viewed from the $j$ th voxel, and gamma rays emitted from voxel $j$ suffer an attenuation loss $\mathrm{A}_{\mathrm{j} k}^{\prime}$ in traveling to voxel $k$. Of those gamma rays that arrive, a fraction given by the scattering cross section $\sigma\left(\theta_{\mathrm{ijk}}\right)$ times the electron density $\eta_{\mathrm{k}}$ in the $k$ th voxel will scatter towards the detector, where $\theta_{i, k}$ is the scattering angle. Of those, a fraction $E_{i k}$ times the attenuation loss $A_{i k}\left(e, \theta_{i j k}\right)_{i k}$ for the scattered gamma rays will be detected (the attenuation depends on the scattered photon energy distribution, which depends on the scattering angle). Overall, we can estimate the scattered detector count rate $\left(d^{\prime}(e)_{\mathrm{i}}\right)_{\mathrm{j} \mathrm{k}}$ due to this particular scattering path as

$$
\left(d^{\prime}(e)_{\mathrm{i}}\right)_{\mathrm{jk}} \cong c_{e} \mathrm{E}_{\mathrm{jk}}^{\prime} \mathrm{A}^{\prime}(e)_{\mathrm{jk}} \mathrm{E}_{\mathrm{ik}} \mathrm{A}\left(e, \theta_{\mathrm{j} \mathrm{k}}\right)_{\mathrm{ik}} \eta_{\mathrm{k}} \sigma\left(e, \theta_{\mathrm{ijk}}\right) s_{\mathrm{j}},
$$

where (as before) $s_{\mathrm{j}}$ is the radionuclide mass in voxel $j$.

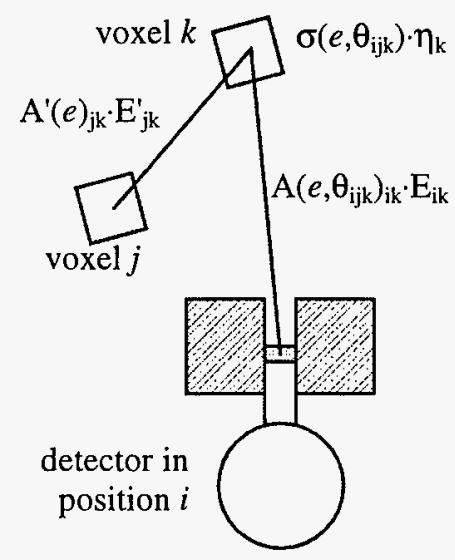

Figure 3: Geometry of the quantities used in the model.

A full implementation of (13) would be cumbersome, so we made some simplifications that significantly reduced the analysis time and computational burden. These included (a) using one energy for scattered gamma rays, (b) computing the $\mathrm{A}^{\prime}(e)_{\mathrm{jk}}$ term from average attenuation coefficients $\mu_{\mathrm{avg}, \mathrm{l}}$ (for layer $l$ ), (c) using one average scattering cross section $\sigma$ for all cases, and (d) limiting the scattering voxels to the layer containing the emitting voxel. With these simplifications, we can estimate the terms in (13) as

$$
\begin{aligned}
& \mathrm{A}\left(e, \theta_{\mathrm{ik} k}\right)_{\mathrm{ik}}=\mathrm{A}_{\mathrm{ik}}(\text { scatter }) \approx\left(\mathrm{F}_{\mathrm{ik}} / \mathrm{E}_{\mathrm{ik}}\right)^{\beta}, \\
& \mathrm{A}^{\prime}(e)_{\mathrm{jk}}=\mathrm{A}_{\mathrm{jk}}^{\prime} \approx \exp \left\{-r_{\mathrm{jk}}^{\prime} \mu_{\mathrm{avg}, \mathrm{l}}\right), \\
& \mathrm{E}_{\mathrm{jk}}^{\prime} \approx\left(1 / r_{\mathrm{jk}}^{\prime}{ }^{2}\right) \cdot \text { constant, }
\end{aligned}
$$

and

$$
\eta_{k}=\Sigma_{z} \alpha_{z} \rho_{z k}
$$

The constant $\beta$ in (14) is the ratio $\mu_{\text {out }} / \mu_{\text {in }}$ for monoenergetic pre- and post-scatter gamma rays, which we estimate to be approximately 1.5 on average for ${ }^{239} \mathrm{Pu}$ spectra. The terms $F_{i k}$ and $E_{i k}$ are the summed gross-count estimates from (10). The term $r_{j k}^{\prime}$ in (15) and (16) is the distance between the centers of voxels $j$ and $k$. The terms $\alpha_{z}$ in (17) are conversion factors required to give the electron densities for the basis materials. Note that (17) is not an approximation. The cross section $\sigma$ is treated as a free parameter; we adjust its value to obtain the best match to actual scan data. This allows us to ignore the calibration terms $c_{\mathrm{e}}$ as well as the constant term in (16).

The total corrected emission matrix $F_{G C}$ is the sum of the scatter-free emission matrix $F$ from (10) and the scatter emission matrix $\mathrm{F}^{\prime}$,

$$
F_{\mathrm{Gc}}=\mathrm{F}+\mathrm{F}^{\prime} \text {, }
$$

where

$$
F_{i j}^{\prime}=\sigma \sum_{k} E_{j k}^{\prime} A_{j k}^{\prime} E_{i k} A_{i k}(\text { scatter }) \eta_{k} .
$$

Equation (19) has been implemented as the gross-count method algorithm in the TGS_FIT [9] image reconstruction software that we use for analyzing TGS scans. The extra computation time needed to add scatter components to the algorithm is roughly the same as that required for adding an extra material to the MBS. For a 266-MHz PC computer, this amounts to about fifteen seconds of extra computing time per assay with the standard 10 by 10 TGS image resolution.

\section{RESULTS AND CONCLUSIONS}

To illustrate the effectiveness of our scatter model we performed a TGS scan of a 100-g metallic low-burnup Pu source embedded in a high-density polyethylene matrix. An HPGe detector was used in the scan so that we could compare results from the usual continuum-subtracted analysis with results from the gross-count method, with and without inclusion of the scattering terms. The source was placed at the outer edge of the imaged area to maximize the number of tomographic views which were beyond the detector's field of view and only scattered gamma rays could be seen. As discussed above, the amount of scatter seen in the data should be greatest with this type of matrix; that is, a fairly dense low$\mathrm{Z}$ matrix, in which many gamma rays are scattered but relatively few are lost before reaching the detector. Figure 4 shows the measured gross-count data for one layer of the scan plotted as count rate versus view index. Also shown are two projections of gross-count scan data made from an image of the source obtained from the $414-\mathrm{keV}$ line in ${ }^{234} \mathrm{Pu}$. One 
projection was made with the scatter-free gross-count matrix $\mathrm{F}$; the other with the full gross-count matrix $F_{\mathrm{GC}}$. The reasoning is that data from the $414-\mathrm{keV}$ scan is free of scattered photon interferences, so the image made from that data should be faithful.

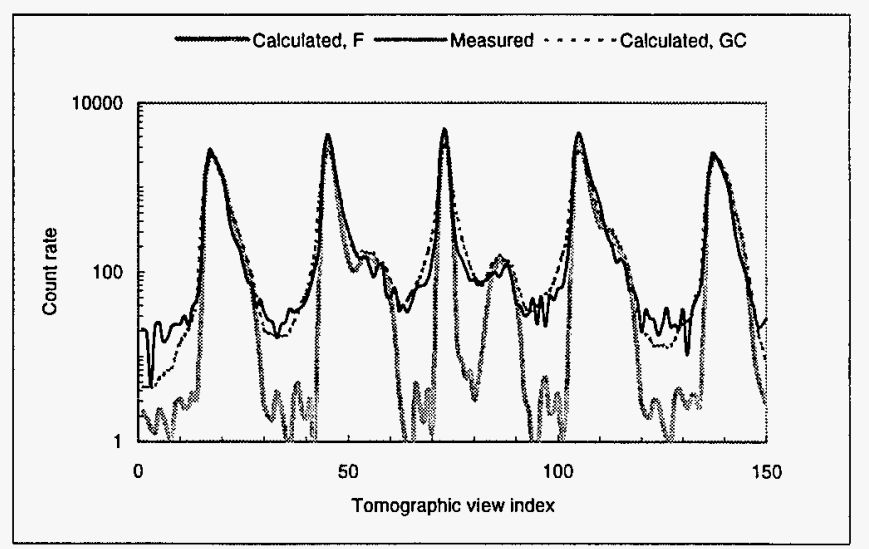

Figure 4: Measured Vs. calculated gross-count scan data for a point $\mathrm{Pu}$ source in a polyethylene matrix. The solid black line shows measured gross-count data. The solid gray line shows projected (simulated) scan data for the same case using the scatter-free matrix $F$. The dashed line shows the projection for the complete matrix, $F_{G C}$, which includes scatter components.

What we see in Figure 4 is that the data simulated from the scatter-free matrix ("Calculated, F" in Figure 4) has the same general appearance as continuum-subtracted data, in that the count rates go to (almost) zero when the source is beyond the detector's field of view. In the measured gross-count data, on the other hand, these "valleys" are partially filled in with counts from the scattered gamma rays. Although the additional counts are a relatively small fraction of the total counts observed, they appear to the image reconstruction routine as distributed sources spread out through the sample volume. Because the attenuation correction needed at the sample's center is many times greater than that needed near the outside of the drum, these excess counts are amplified in the final mass estimate. For this particular case, the mass estimated from the gross count scan without the scatter correction was 1.35 times the correct value, even though the scattered gamma rays added only $7 \%$ to the total rate. The data simulated using the full gross count matrix, ("Calculated, GC" in Figure 4 ) on the other hand, simulates very well the scattered photon contribution, as can be seen in the figure. The use of the full gross-count matrix $F_{G C}$ in analyzing the scan data resulted in a much better image of the source (less noise) and a correspondingly better mass estimate of 0.97 times the correct value.

Our results at this stage are very encouraging, but further investigation is needed. We need to take additional data with a wide range of matrix types and source distributions before we can conclude that the simple assumptions used in developing our scatter model are justified.

\section{REFERENCES}

[1] R. J. Estep, T. H. Prettyman, and G. A. Sheppard, "Tomographic Gamma Scanning to Assay Heterogeneous Radioactive Waste," Nuclear Science and Engineering, 118, 1994, p. 145-152.

[2] R. J. Estep, S. Melton, "Using NaI Detectors for Tomographic Gamma Scanning," Los Alamos National Laboratory, LA-UR-97-56, 1997.

[3] R. J. Estep, T.H. Prettyman, and G. S. Sheppard, "Reduction of TGS Image Reconstruction Times Using Separable Attenuation Coefficient Models," Los Alamos National Laboratory, LA-UR-95-2013, Proc. American Nuclear Society Winter Meeting, San Francisco, CA, 1995.

[4] P. Engler, W. D. Friedman, and E. E. Armstrong, "Determination of Material Composition Using Dual Energy Computed Tomography on a Medical Scanner," ASNT Topical Proc. Conf. On Industrial Computerized Tomography, pp. 142-146.

[5] R. J. Estep, T. H. Prettyman, and G. A. Sheppard, "Comparison of Attenuation Correction Methods for TGS and SGS: Do we really need selenium-75?" Proc. $37^{\text {th }}$ annual Meeting, Naples, FL (July, 1996)

[6] R. J. Estep, M. W. Rawool-Sullivan, D. Miko, "A Method for Correcting NaI Spectra for Attenuation Losses in HandHeld Instrument Applications," presented at this conference.

[7] W. R. Leo, "Techniques for Nuclear and Particle Physics Experiments: A How-to Approach," Second Edition, Berlin:Springer-Verlag, 1994, p. 56

[8] R. J. Estep, "TGS-FIT: Image Reconstruction Software for Quantitative, Low-Resolution Tomographic Assays," Los Alamos National Laboratory, LA-12497-MS, 1993. 

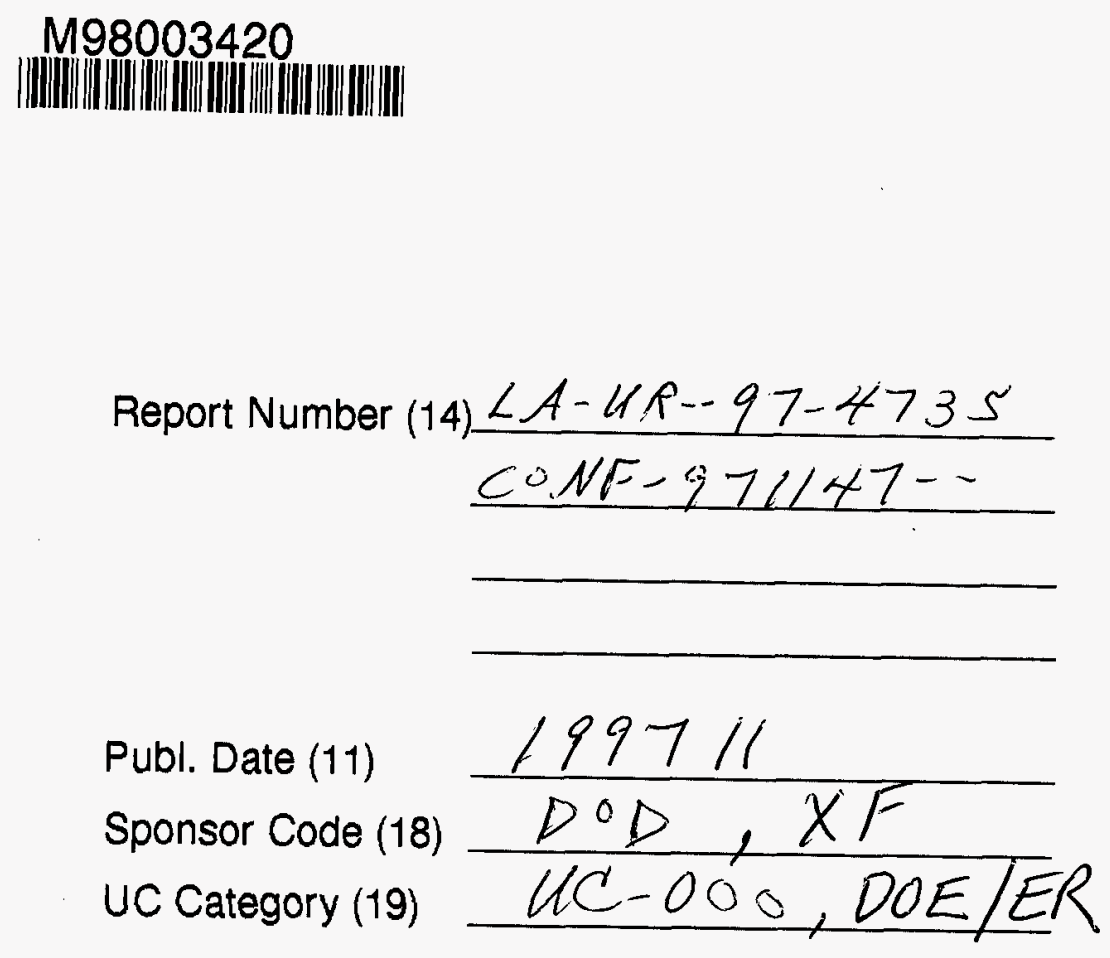